\title{
Exeunt Lateral Decubitus Radiographs
}

\author{
Jane A. Shaw Coenraad F.N. Koegelenberg \\ Division of Pulmonology, Department of Medicine, Tygerberg Academic Hospital, Stellenbosch University, \\ Cape Town, South Africa
}

In 1924, in a stroke of ingenuity belied by its simplicity, Merlo Gomez and Heidenreich decided to turn individuals with suspected small pleural effusions into the lateral decubitus position [1]. What they described was the closest thing to a dynamic imaging technique that the era could offer, and they used it admirably. Between themselves and several subsequent investigators, they demonstrated that pleural fluid volumes as small as $10 \mathrm{~mL}$ could be identified on a lateral decubitus chest radiograph. These tiny volumes would certainly have been invisible to even the most experienced viewer of the erect radiograph, which requires $50 \mathrm{~mL}$ accumulated fluid on a standard lateral erect film and $200 \mathrm{~mL}$ on a posteroanterior erect film in order to become visible [2]. In addition to the detection of pleural effusions that otherwise might have been missed, the champions of the lateral decubitus radiograph have used it for the quantification of pleural fluid volume - which may prove relevant in determining if intervention is warranted in parapneumonic effusions [3] - and the identification of loculated effusions. Sadly, it would appear that these heroes of initiative - these creative giants! - have now had their share of the limelight and must cede the stage to others.

Enter thoracic ultrasound (TUS). The origin story of this superstar spans over 2 centuries, from the study of echolocation in bats in the late 1700s, to the first medical use of ultrasound to assess brain tumors in the mid-1900s, to the current permeation of this imaging technology

\section{KARGER}

() 2018 S. Karger AG, Basel

E-Mail karger@karger.com

www.karger.com/res throughout almost all clinical fields. In interventional pulmonology it is considered the standard of care to use TUS to plan procedures and interventions, as it allows for a safe site selection and identification of the intercostal vessels. This has the benefit of reducing the incidence of complications, as well as allowing swift identification and thereby early management - of iatrogenic injury [4]. There is evidence that TUS is more accurate than the lateral decubitus at determining the volume of fluid in a clinically significant effusion, and it is clear that TUS is superior to conventional radiography and computed tomography at identifying loculations $[1,4]$.

In this issue of Respiration, Thiam et al. have contributed to the data on identification of pleural fluid loculations via the lateral decubitus radiograph [5]. The utility of identifying loculated effusions is not in question: it is acknowledged that a loculated effusion is more likely to progress to empyema, has prognostic implications, and predicts the success or failure of the chosen intervention. Nor is the best method of identifying loculations in question: it is acknowledged to be the TUS. Rather, the authors propose that in certain areas of the world, resources are limited to such an extent that an ultrasound machine (or the expertise to use it) may not be readily available prior to pleural procedures, and thus they set out to assess whether the lateral decubitus radiograph - which is cheap and almost universally available - might provide a viable alternative. The answer they discovered was a resounding 
negative. Within their database, they collected information from 86 patients who had had a medical thoracoscopy with documentation of the presence or absence of pleural adhesions - considered as the gold standard. They independently collected the lateral decubitus radiographs of these patients and assessed for the presence or absence of the 2 signs associated with loculated effusions: an incomplete horizontal fluid level or an irregular horizontal fluid level. Their data generated a sensitivity, specificity, positive predictive value, and negative predictive value of $71.2,44.1,66.1$, and $50 \%$, respectively, for the shape of the fluid level on the lateral decubitus radiograph's ability to predict underlying pleural adhesions. The overall diagnostic accuracy of the "incomplete horizontal fluid level" was $60.5 \%$, and of the "irregular horizontal fluid level" it was $53.5 \%$. The conclusion drawn by the authors was that clinicians should be skilled in ultrasonography techniques and that TUS become more widely available, especially in areas with high rates of pleural effusions that are likely to have loculations, such as tuberculous pleural effusions.

It should be highlighted that TUS is not as prohibitively expensive as is postulated in the authors [6]. Firstly, an ultrasound machine is useful across many clinical disciplines, as well as being portable: even an entry-level machine with no Doppler or M-mode and with a single curvilinear probe contributes in significant ways to many different disciplines, including emergency medicine, obstetrics and gynecology, nephrology, and gastroenterology to name but a few [6]. Where all benefit, all can share the cost. Furthermore, there are no consumables involved other than those of the procedure itself. An ultrasound machine that is well cared for can be useful for a decade or more, so it represents a once-off expenditure, far cheaper than a computed tomography scan. Additionally, one saves the cost of managing the procedural complications that are avoided by using ultrasound guidance. TUS has also been shown to be an excellent guide for transthoracic pulmonary, pleural, and mediastinal biopsy [7]. Moreover, the use of TUS decreases the need for more invasive and far more expensive investigations: TUSguided pleural biopsy decreases the need for medical thoracoscopy by $90 \%$, and TUS-guided mediastinal biopsy can decrease the need for mediastinoscopy by $93 \%[8,9]$. Ultrasound-guided pulmonary biopsy of masses that abut the chest wall has a sensitivity equal to CT-guided biopsy, with the advantage of fewer pneumothoraces, a shorter procedure time, and no irradiation [10].

With regards to skill acquisition, it seems to us that the need has arisen to introduce a familiarity with the ultrasound machine and its interpretation in basic medical training and for clinicians to attain at least basic (level 1) competency. By no means should this technique replace a clinical examination; however, its utility in the general daily practice of so many areas of medicine is undisputed, and its use is now so commonplace that a medical graduate who is not familiar with the ultrasound is unfortunately behind the curve.

Certainly, as the study by Thiam et al. confirms, where the identification of loculated pleural effusions is concerned, it is time for the lateral decubitus to make a long overdue graceful exit, and for TUS to take center stage. All clinicians involved in thoracic medicine, whether in the developed or developing world, should therefore have basic TUS competency.

\section{References}

1 Metersky ML: Is the lateral decubitus radiograph necessary for the management of a parapneumonic pleural effusion? Chest 2003; 124:1129-1132.

-2 Soni NJ, Franco R, Velez MI, Daniel Schnobrich, Dancel R, Restrepo MI, Mayo PH: Ultrasound in the diagnosis and management of pleural effusions. J Hosp Med 2015;10:811816.

>3 Light RW, Girard WM, Jenkinson SG, George RB. Parapneumonic effusions. Am J Med 1980;69:507-512.

4 Corcoran JP, Tazi-Mezalek R, Maldonado F, Yarmus LB, Annema JT, Koegelenberg CFN, et al: State of the art thoracic ultrasound: intervention and therapeutics. Thorax 2017;72: 840-849.
5 Thiam K, Berbis J, Laroumagne S, Guinde J, Chollet B, Dutau H: Diagnostic accuracy of lateral decubitus chest radiography before pleural maneuvers for the management of pleurisies at the era of chest ultrasound. Respiration 2018, DOI: 10.1159/000487999

-6 Koegelenberg CFN, Von Groote-Bidlingmaier F, Bolliger CT: Transthoracic ultrasonography for the respiratory physician. Respiration 2012;84:337-350.

7 Corcoran JP, Hew M, Maldonado F, Koegelenberg CF: Ultrasound-guided procedures; in Laursen CB, Rahman NM, Volpicelli G (eds): Thoracic Ultrasound (ERS Monograph). Sheffield, European Respiratory Society, 2018, pp 226-243.
$>8$ Koegelenberg CF, Irusen EM, von GrooteBidlingmaier F, Bruwer JW, Batubara EM, Diacon AH: The utility of ultrasound-guided thoracocentesis and pleural biopsy in undiagnosed pleural exudates. Thorax 2015;70:995957

-9 Koegelenberg CF, Diacon AH, Irusen EM, von Groote-Bidlingmaier F, Mowlana A, Wright CA, Louw M, Schubert PT, Bolliger CT: The diagnostic yield and safety of ultrasound-assisted transthoracic biopsy of mediastinal masses. Respiration 2011;81:134-141.

10 Sconfienza LM, Mauri G, Grossi F, et al: Pleural and peripheral lung lesions: comparison of US- and CT-guided biopsy. Radiology 2013; 266:930-935. 